Research Article

\title{
Discrimination and Correlation Analysis of Multiview SAR Images with Application to Target Recognition
}

\author{
Lin Chen, ${ }^{1}$ Peng Zhan, ${ }^{1}$ Luhui Cao, ${ }^{2}$ and Xueqing $\mathrm{Li} \mathbb{D}^{1}$ \\ ${ }^{1}$ School of Software, Shandong University, Jinan 250100, China \\ ${ }^{2}$ Information Office, Shandong University, Jinan 250100, China \\ Correspondence should be addressed to Xueqing Li; autuju@yeah.net
}

Received 30 December 2020; Revised 8 February 2021; Accepted 18 February 2021; Published 26 February 2021

Academic Editor: Mu-Chen Chen

Copyright (c) 2021 Lin Chen et al. This is an open access article distributed under the Creative Commons Attribution License, which permits unrestricted use, distribution, and reproduction in any medium, provided the original work is properly cited.

A multiview synthetic aperture radar (SAR) target recognition with discrimination and correlation analysis is proposed in this study. The multiple views are first prescreened by a support vector machine (SVM) to select out those highly discriminative ones. These views are then clustered into several view sets, in which images share high correlations. The joint sparse representation (JSR) is adopted to classify SAR images in each view set, and all the decisions from different view sets are fused using a linear weighting strategy. The proposed method makes more sufficient analysis of the multiview SAR images so the recognition performance can be effectively enhanced. To test the proposed method, experiments are set up based on the moving and stationary target acquisition and recognition (MSTAR) dataset. The results show that the proposed method could achieve superior performance under different situations over some compared methods.

\section{Introduction}

As one of the classical problems in remote sensing pattern recognition field, synthetic aperture radar (SAR) target recognition has been researched for decades [1]. Previously, a SAR target recognition algorithm was usually conducted on a single-view SAR image via feature extraction and classification processes. Discriminative features are first extracted from SAR images, e.g., geometrical properties, scattering characteristics, or transformation features. In $[2,3]$ the geometrical features were used for SAR target recognition like target region and contour. The attributed scattering centers were adopted as the basic features in $[4,5]$, which were matched for target recognition. A variety of transformation features have been employed in SAR target recognition, e.g., principal component analysis (PCA) [6], non-negative matrix factorization (NMF) [7], monogenic signals $[8,9]$, etc. The classifiers aim to dig out the discriminability in the extracted features thus reaching correct decisions on the test samples. As a witness to the progress in pattern recognition techniques, a rich set of classifiers were successfully used in SAR target recognition including $K$ - nearest neighbor (KNN) [6], support vector machine (SVM) [10], and adaptive boosting (AdaBoost) [11]. Based on the compressive sensing theory, the sparse representation-based classification (SRC) was developed with application to face recognition [12], SAR target recognition [13, 14], etc. Recently, with the rapid development of deep learning algorithms, some convolutional neural network- (CNN-) based SAR target recognition methods were proposed $[15,16]$.

Nowadays, the data acquisition capability of SAR sensors is greatly enhanced. Then, the multiview SAR images of the same target are available, which could be jointly used to improve the recognition performance. A few multiview SAR target recognitions have been already developed with much superior performance over single-view ones. Brown designed a multiview decision fusion algorithm for multiview SAR images based on Bayesian theory [17]. In [18], a voting strategy for the decisions from multiple views by SVM was developed. Zhang et al. first applied joint sparse representation (JSR) to the classification of multiview SAR images, which intended to exploit the inner correlations between different views [19]. JSR is a general extension of traditional SRC to multitask learning, which is capable of 
representing each task precisely under the correlation constraints among different tasks. Inspired by the good performance of JSR, several modifications of the work in [19] were further proposed by improving the dictionary or classification scheme [20, 21]. Some issues need to be highlighted in multiview SAR target recognition method. First, as mentioned in [21], because of the extended operating conditions (EOCs), not all of the multiple views are discriminative enough for correct decisions. Then, it is preferred that those with low discrimination were rejected before the classification stage. Second, the multiview SAR images may be from quite different azimuths. Due to the azimuthal sensitivity, the multiview SAR images may be costly related or relatively independent in terms of the azimuthal differences. In this case, the direct use of JSR may bring some incorrect constraints. Based on the above considerations, this study proposes a multiview SAR target recognition method via discrimination and correlation analysis of multiple views. First, SVM is used as the classifier to perform discrimination analysis. Each of the multiview SAR images is first classified by SVM. Based on the output posterior probabilities, a decision reliability is defined, which can effectively evaluate the classification precision of the present view. Afterwards, a thresholding judgement is conducted to reject those views with lower decision reliabilities in comparison with the preset threshold. For the selected views, a clustering algorithm is designed to cluster them into several independent view sets. It is assumed that views in the same set share high correlations while different view sets are relatively independent. Then, for each set, JSR is employed to classify all the views in it to exploit their correlations. A linear weighting strategy is adopted to fuse the decisions from different view sets and the fused decision values are used to decide the target label. The main contributions of this paper are as follows. First, the proposed method comprehensively considers the discrimination and correlation in the multiview SAR images. Therefore, it is promising that the multiple views can be exploited fully to enhance the recognition performance. Second, two classifiers, i.e., SVM and JSR, are combined in a joint recognition framework. They two classifiers are both effective for SAR target recognition and have complementary merits. So, their combination could probably help improve the recognition performance. To validate the performance of the proposed method, experiments are set up on the moving and stationary target acquisition and recognition (MSTAR) dataset under different situations. The experimental results confirm the high effectiveness and robustness of the proposed method.

\section{SVM-Based Discrimination Analysis}

SVM is chosen as the basic classifier for discrimination analysis [10], which aims to find out those highly discriminative views while rejecting those indiscriminative ones. Since the proposal by Vapnik et al. in 1995, SVM has long been a hot classifier in pattern recognition field. In 2001, Zhao and Principe first introduced SVM into the field of SAR target recognition. Afterwards, it was widely used to classify different types of features as reported in [2, 3, 7]. With the objective to minimize the structural risk, SVM is able to find a hyperplane to separate two different patterns. Then, for an unknown sample $x$, the decision function is as follows:

$$
f(x)=\sum_{i=1}^{M} w_{i} y_{i} K\left(x_{i}, x\right)+b, \quad \alpha_{i} \geq 0, \forall i
$$

where $x_{i}(i=1, \ldots, M)$ represents the support vectors drawn from the training samples; $y_{i}= \pm 1$ denote the two pattern labels; $w_{i}(i=1, \ldots, M)$ and $b$ are the parameters to be estimated, i.e., weights and bias; and $K(\cdot)$ is the kernel function, which can be properly designed to enhance the nonlinear classification capability. Some common kernel functions are linear kernel, polynomial kernel, radial basis function (RBF) kernel, etc.

Traditionally, SVM was designed for two-class separation task. In order to handle multiclass classification problems, the multiclass SVM was developed using oneversus-one or one-versus-rest strategies as we can find these functions in the famous LIBSVM toolbox.

In this study, each of the multiview SAR mages is first classified by SVM for prescreening. There are $C$ classes of training samples. For a single view, the posterior probabilities output from SVM corresponding to different classes are denoted as $\left[p_{1}, p_{2}, \ldots, p_{C}\right]$. The decision reliability is defined as follows:

$$
R=\frac{\max \left(p_{j}\right)}{p_{k}}, \quad(j \neq k),
$$

where $p_{k}$ denotes the maximum in the $C$ probabilities. So, the decision reliability $R$ is a constant in the range of $[0,1]$. A lower $R$ indicates a more reliable decision because the maximum probability is notably larger than the remaining ones. On the contrary, a high $R$ infers a decision not reliable enough. With an appropriate threshold $T_{1}$, those views with larger decision reliabilities than $T_{1}$ are rejected. And the remaining views are assumed to be highly beneficial for the multiview target recognition, which are used in the following classification stage.

\section{Correlation Analysis}

3.1. Clustering of Multiview SAR Images. After the discrimination analysis by SVM, only $M$ of total $N$ views are selected for the following classification. However, due to azimuthal sensitivity of SAR images, the selected views may not be closely related. When two images are from approaching azimuths, they share high correlations. Otherwise, when their azimuths are notably different, they have a low similarity. Hence, it is necessary to analyze the relationship among the multiview SAR images thus designing a suitable classification scheme.

In this paper, a clustering algorithm of multiview SAR images is developed based on the correlation of two images. The correlation coefficient between two SAR images is defined as follows: 


$$
c_{i j}=\max \left(\frac{\sum_{k} \sum_{l}\left[I_{i}(k, l)-m_{1}\right]\left[I_{j}(k-\Delta k, l-\Delta l)-m_{2}\right]}{\left[\sum_{k} \sum_{l}\left[I_{i}(k, l)-m_{1}\right]^{2}\left[I_{j}(k-\Delta k, l-\Delta l)-m_{2}\right]^{2}\right]^{1 / 2}}\right) .
$$

In equation (3), $m_{1}$ and $m_{2}$ represent the mean values of the two images: $I_{i}$ and $I_{j}$, respectively. When $I_{i}$ slides on $I_{j}$, the maximum correlation coefficient is defined as the final correlation coefficient.

Denote the $M$ selected multiview SAR images as $V=\left\{I_{1}, I_{2}, \ldots, I_{M}\right\}$. At first, the correlation coefficients between each pair of views are calculated as a matrix in Table 1. Afterwards, the designed clustering algorithm is performed as the following steps:

Step 1. Choose $I_{1}$ as the cluster center

Step 2. Select the correlation coefficients in the first row of $C$ lower than $T_{2}$

Step 3. Obtain the first cluster, which comprises $I_{1}$ and those from Step 2

Step 4. Remove views in the first cluster from $V$; repeat Step 1 to Step 3 to obtain the next cluster

Step 5. Repeat Step 1 to Step 4 until each view are included in a single cluster

After the clustering algorithm, the multiview SAR images are included into several view sets. In each view set, SAR images are assumed to share high correlations related to the threshold $T_{2}$. On the contrary, different view sets are assumed to be relatively independent.

3.2. JSR of Multiview SAR Images. In each view set after the clustering, the included SAR images share high correlations. In this case, it is preferred that JSR can be used to jointly represent the multiview SAR images with the constraints of their correlations. Denote the $P$ views in a view set as $Y=\left[y^{(1)}, \ldots, y^{(P)}\right]$; their sparse representation problems can be considered together as follows:

$$
\min _{A}\left\{g(A)=\sum_{m=1}^{P}\left\|y^{(m)}-X \alpha^{(m)}\right\|\right\}
$$

where $X$ represents the global dictionary established by all the training samples and $A=\left[\alpha^{(1)}, \ldots, \alpha^{(P)}\right]$ comprises the sparse coefficient vectors from different views. However, the problem in equation (4) considers different views independently. In order to properly use the inner correlations among them, the $\ell_{1,2}$ mixed norm is usually imposed on the coefficient matrix $A$ to formulate an optimization objective as follows:

$$
\min _{A} g(A)+\lambda\|A\|_{1,2}
$$

TABLE 1: Correlation coefficient matrix of multiview SAR images.

\begin{tabular}{ccccc}
\hline & $I_{1}$ & $I_{2}$ & $\cdots$ & $I_{M}$ \\
\hline$I_{1}$ & $c_{11}$ & $c_{12}$ & $\cdots$ & $c_{2 M}$ \\
$I_{2}$ & $c_{21}$ & $c_{22}$ & $\cdots$ & $c_{2 M}$ \\
$\vdots$ & $\vdots$ & $\vdots$ & $\ddots$ & $\vdots$ \\
$I_{M}$ & $c_{M 1}$ & $c_{M 2}$ & $\cdots$ & $c_{M M}$ \\
\hline
\end{tabular}

where $\|A\|_{1,2}$ returns the $\ell_{1,2}$ mixed norm of $A$ by calculating $\ell_{2}$ norm of each row in $A$ and $\ell_{1}$ of the resulting vector afterwards.

Owing to the progress in compressive sensing theory, there are algorithms like simultaneous orthogonal matching pursuit (SOMP) [22] and multitask Bayesian compressive sensing (BCS) [23], which can be used to solve the optimization problem in equation (5). Based on the solved coefficient matrix, the total reconstruction error of all the views by a single training class can be calculated as follows:

$$
r_{i}=\sum_{m=1}^{P}\left\|y^{(m)}-X_{i}^{(m)} \alpha_{i}^{(m)}\right\|_{2}
$$

where $r_{i}$ denotes the total reconstruction error from the $i$ th training class. Traditionally, by comparing the reconstruction errors from different training classes, the target label of the test sample $(s)$ is assigned to the one with the minimum error. Specifically in this study, there are several view sets, which are represented by JSR, respectively. Therefore, a proper fusion of their results can help improve the precision of the final decision.

3.3. Linear Weighting. There are $K$ view sets after the clustering. For the $k$ th view set, the output reconstruction errors from JSR are $\left[r_{1}^{k}, r_{2}^{k}, \ldots, r_{C}^{k}\right]$. This study uses a linear weighting strategy to fuse the reconstruction errors from different view sets as follows:

$$
\mathrm{fr}_{i}=\omega_{1} r_{i}^{1}+\omega_{2} r_{i}^{2}+\cdots+\omega_{K} r_{i}^{k}
$$

where $\mathrm{fr}_{i}$ denotes the fused reconstruction error to the $i$ th training class and $\omega_{1}, \omega_{2}, \ldots, \omega_{K}$ are the linear weights of different view sets subject to

$$
w_{0}+w_{1}+\cdots+w_{n}=1 .
$$

In this study, the weight of a view set is decided based on the number of views in it. For example, if there are $M_{k}$ views in the $k$ th view set, the corresponding weight is calculated as $\omega_{k}=\left(M_{k} / M\right)$. It is assumed that when a view set includes more images, it contains more information for correct decision so a higher weight is assigned.

Figure 1 illustrates the implementation details of the proposed multiview SAR target recognition method. The training samples are used to support the two classifiers, i.e., SVM and JSR. Considering the high dimension of original SAR images, the random projection algorithm [13] is used to reduce the training and multiview test samples to 520-dimension feature vectors for classification. 


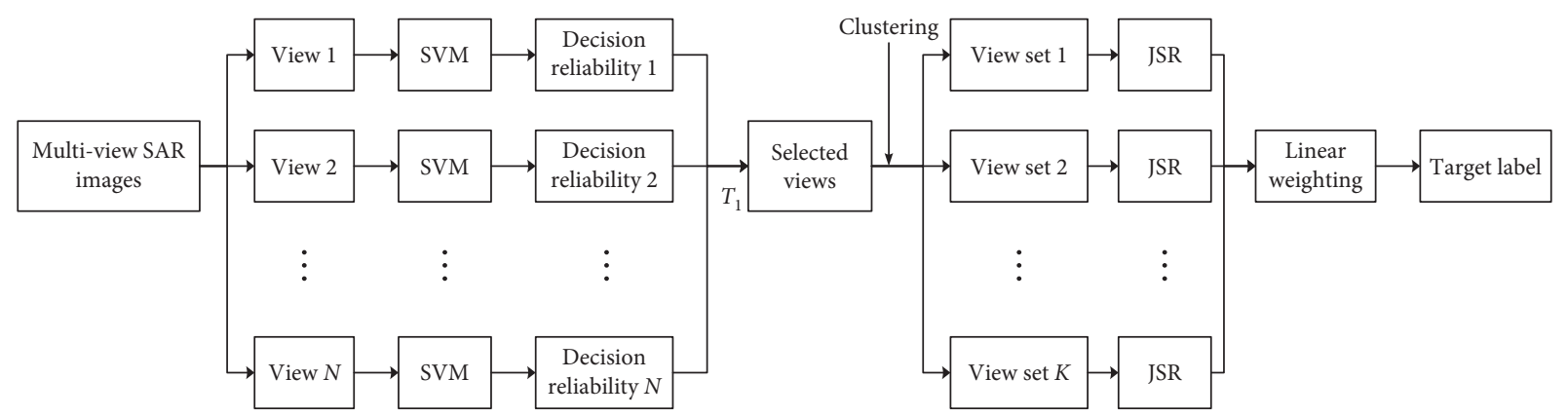

FIGURE 1: Implementation of the proposed multiview SAR target recognition method.

Figure 2 further explains the proposed method with a concrete example with MSTAR SAR images. There are six views of the same target with different azimuths to be classified indexed as " 1 " to " 6 " sequentially. First, those views are classified based on SVM and their reliability levels are calculated to be $0.53,0.42,0.27,0.39,0.57$, and 0.36 , respectively. With a threshold of 0.3 , the view indexed 3 is unselected while the remaining ones are used in the following stages. Using the clustering algorithm, the five views are reorganized into two subsets with indexes as $\{1,6,7\}$ and $\{2,4,5\}$. Afterwards, the two subsets are independently processed by JSR to obtain the corresponding reconstruction error vectors, which are fused to make the final decision.

\section{Experiments and Analysis}

4.1. Dataset and Compared Methods. MSTAR dataset is chosen as the data source to validate the performance of this method. In the dataset, a large volume of SAR images collected from ten ground targets (shown in Figure 3) are available for training and testing. All the images have high resolutions of $0.3 \mathrm{~m}$ at both range and cross range directions. Typical experimental situations including the standard operating condition (SOC) and EOCs can be set up to comprehensively evaluate the proposed method.

Simultaneously in each experiment, some single-view and multiview SAR target recognition methods from previous literatures are compared. The single-view methods are SVM from [10], SRC from [13], and CNN from [15]. The multiview methods are $[18,19]$, respectively, which are denoted as MultiView1 and MultiView2. For simplicity, ten views with azimuth step of $3^{\circ}$ are used for the three multiview methods. In the following, the proposed method is first tested under SOC to validate its preliminary performance and obtain the best parameters for recognition. Later, some typical EOCs, e.g., configuration variance, depression angle variance, and noise corruption, are set up to evaluate the robustness of the proposed method.

4.2. Recognition under SOC. SOC is a simple situation in SAR target recognition, under which the test samples are similar to the training ones. Table 2 gives the training and test sets under SOC. Images at $17^{\circ}$ depression angle are trained to classify those from $15^{\circ}$. At first, the two thresholds
$T_{1}$ and $T_{2}$ are set to be 0.3 and 0.4 , respectively. Figure 4 displays the confusion matrix of the proposed method, where the recognition rate of each of the ten targets can be observed on the diagonal. The average recognition rates (ARRs) of different methods are summarized in Table 3 for comparison. Clearly, the multiview methods generally achieve much higher ARRs than those of single-view ones because more discrimination is available in multiview SAR images. The proposed method has the highest ARR, validating its best effectiveness under SOC. Compared with another two multiview methods, the proposed method enhances the recognition performance to some extent due to the comprehensive analysis in this study. However, because of the simplicity of SOC, other methods could also achieve very high ARRs so the improvement in this method is not remarkable enough. By varying the two thresholds, the ARRs of the proposed method at different parameters are listed in Table 4. It is noticeable that the two thresholds have influences on the final recognition performance because they affected the preselection of the multiple views as well as the clustering of the views. In comparison, the highest ARR occurs at $T_{1}=0.3$ and $T_{2}=0.4$, which is the reason of setting the two thresholds in the former test. In the following tests, the two thresholds keep unchanged for convenience.

4.3. Recognition under EOCs. EOCs are common in SAR target recognition because of the variations of targets' structures, environment, or radar sensors. In is urgent that SAR target recognition could well handle different types of EOCs thus improving the practicability in real-world scenarios.

4.3.1. Configuration Variance. Caused by the variations of targets' structures, there exist configuration variances in the same types of targets. Table 5 displays the training and test sets under configuration variance, in which the configuration variances occur in BMP2 and T72. Classifying these test samples by all the methods, their ARRs are listed in Table 6. Due to the configuration variances, the ARRs of all the methods decrease disproportionately. In comparison, the proposed one still achieves the highest ARR, validating its higher robustness to possible configuration variances. The prescreening via SVM and clustering algorithm can 


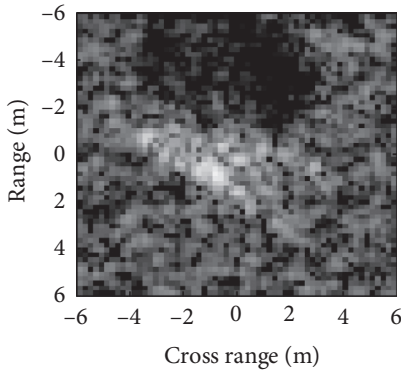

(a)

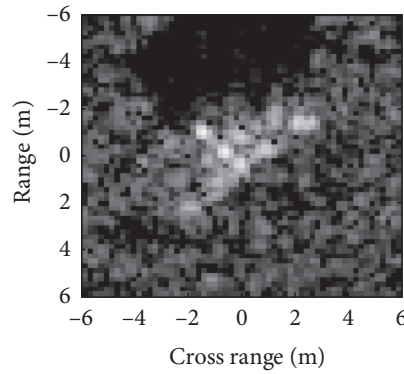

(b)

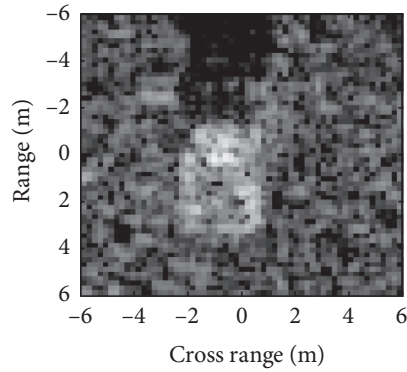

(c)

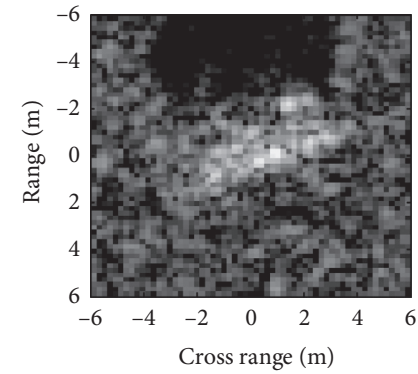

(d)

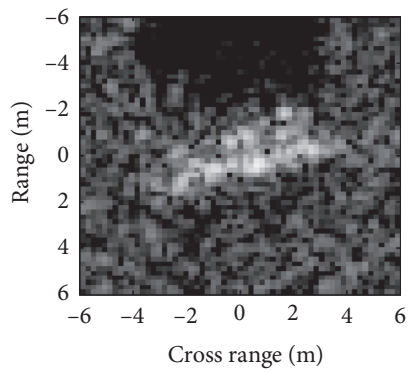

(e)

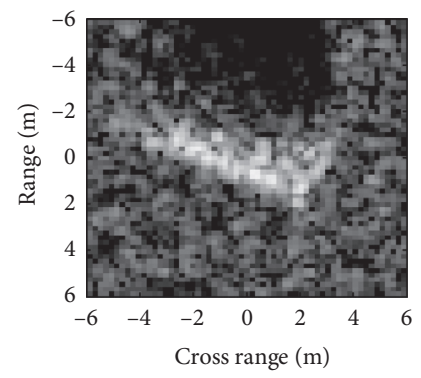

(f)

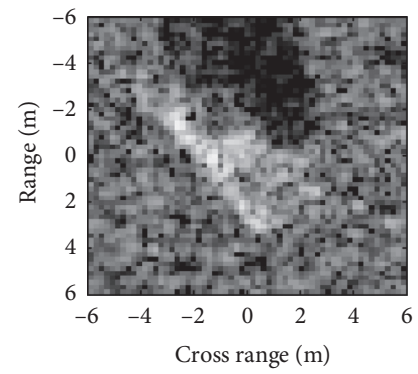

(g)

FiguRe 2: Illustration of multiview SAR images from different azimuths. (a) View l. (b) View 2. (c) View 3. (d) View 4. (e) View 5. (f) View 6. (g) View 7.

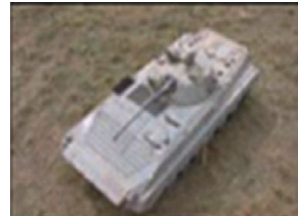

(1) BMP2

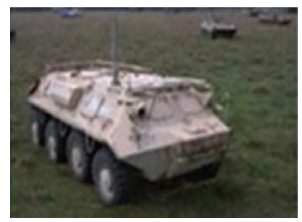

(6) BTR60

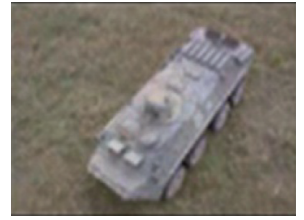

(2) BTR70

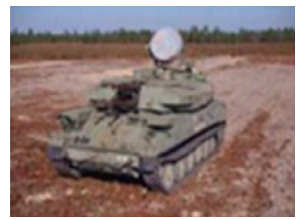

(7) ZSU23/4

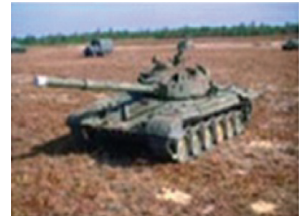

(3) T72

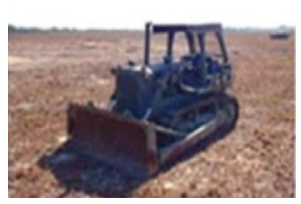

(8) D7

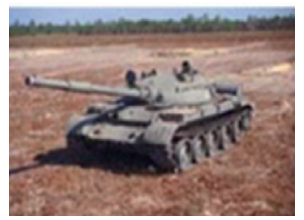

(4) T62

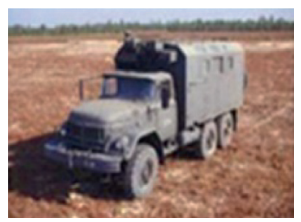

(9) ZIL131

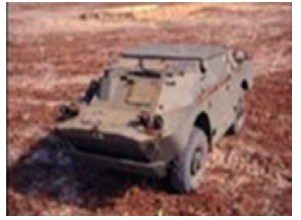

(5) BRDM2

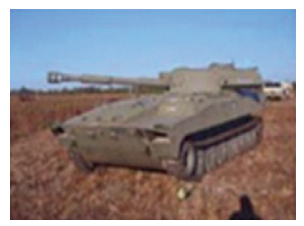

(10) $2 \mathrm{~S} 1$

FIgURE 3: Optical images of the ten MSTAR targets.

effectively select those views beneficial for target recognition. Therefore, the multiview recognition performance could be enhanced as compared with another two multiview methods.

4.3.2. Depression Angle Variance. When the relative positions between the target and radar change, the depression angle for SAR imaging may change simultaneously. However, SAR images of the same target from different depression angles tend to have many divergences even at the same azimuths. As a result, the recognition problems under depression angle variance become much more difficult than SOC. Table 7 gives the training and test sets for this experiment, in which the depression angles occur in 2S1, BDRM2, and ZSU23/4.
Figure 5 summarizes the ARRs of different methods at $30^{\circ}$ and $45^{\circ}$ depression angles, respectively. In comparison, the proposed method defeats the compared ones at both depression angles, reflecting its robustness to possible depression angle variances.

4.3.3. Noise Corruption. Due to the noises from the realworld environment and radar equipment, the test samples to be classified may have a much lower signal-tonoise ratio (SNR) than that of training samples. As a simulation, different levels of Gaussian noises $[24,25]$ are first added to the test samples in Table 2. Figure 6 illustrates the simulated noisy SAR images at different levels, in which the target characteristics are blurred by the noises especially at low SNRs. Afterwards, those noisy 
TABLE 2: Training and test sets used under SOC.

\begin{tabular}{lccr}
\hline \multirow{2}{*}{ Class } & \multicolumn{2}{c}{ Training set } & \multicolumn{2}{c}{ Test set } \\
& Depression angle & Number of samples & Depression angle \\
BMP2 & & 233 & 195 \\
BTR70 & 233 & 196 \\
T72 & 232 & 196 \\
T62 & & 299 & 273 \\
BDRM2 & 298 & $15^{\circ}$ & 274 \\
BTR60 & $17^{\circ}$ & 256 & 195 \\
ZSU23/4 & 299 & 274 \\
D7 & & 299 & 274 \\
ZIL131 & 299 & 274 \\
2S1 & 299 & 274 \\
\hline
\end{tabular}

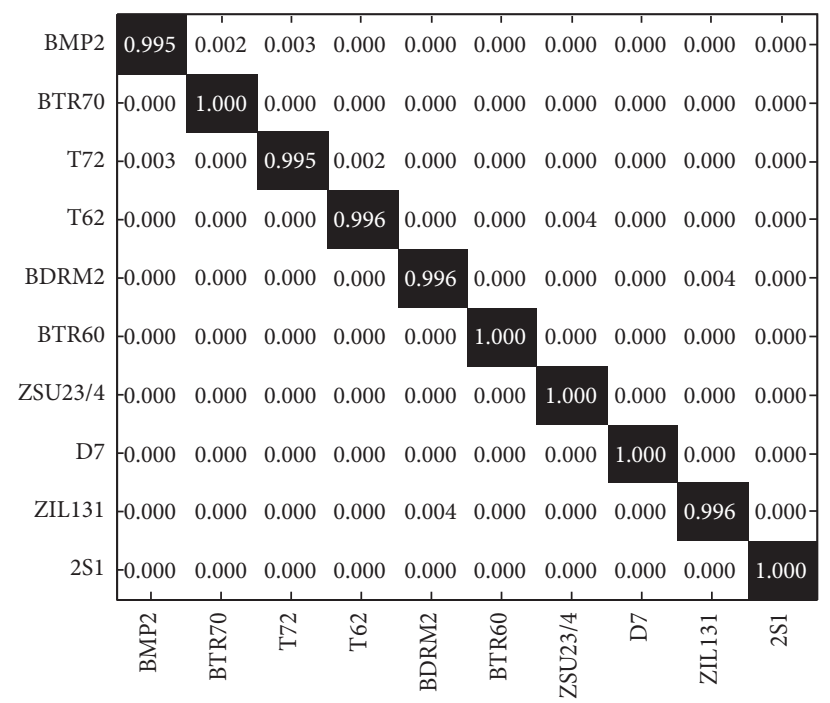

Figure 4: Confusion matrix under SOC.

TABLE 3: Comparison of ARRs under SOC.

\begin{tabular}{lcccccc}
\hline Method & Proposed & SVM & SRC & CNN & MultiView1 & MultiView2 \\
\hline ARR (\%) & 99.58 & 97.04 & 96.28 & 99.06 & 99.13 & 99.32 \\
\hline
\end{tabular}

TABLE 4: ARRs of the proposed method at different combinations of the two thresholds.

\begin{tabular}{|c|c|c|c|c|c|c|}
\hline \multirow[b]{2}{*}{$T 1$} & \multicolumn{6}{|c|}{$T 2$} \\
\hline & 0.1 & 0.2 & 0.3 & 0.4 & 0.5 & 0.6 \\
\hline 0.1 & 98.62 & 98.94 & 99.08 & 99.04 & 98.73 & 98.32 \\
\hline 0.2 & 98.84 & 99.03 & 99.14 & 99.10 & 98.92 & 98.56 \\
\hline 0.3 & 98.97 & 99.06 & 99.31 & 99.18 & 99.04 & 98.79 \\
\hline 0.4 & 99.14 & 99.35 & 99.58 & 99.27 & 99.10 & 99.02 \\
\hline 0.5 & 99.16 & 99.28 & 99.47 & 99.31 & 99.16 & 99.08 \\
\hline 0.6 & 99.10 & 99.21 & 99.38 & 99.27 & 99.12 & 99.04 \\
\hline
\end{tabular}

samples are classified by different methods and their ARR curves are shown in Figure 7. The ARRs of the proposed method top at each SNR, showing its superior robustness to noise corruption over the compared ones. Both the SVM prescreening and clustering could help analyze the multiview SAR images aiming to improve the recognition performance under noise corruption. Therefore, the proposed method could work more robustly in this situation as compared with other single-view and multiview methods. 
TABLE 5: Training and test sets under configuration variance.

\begin{tabular}{lcccc}
\hline \multirow{2}{*}{ Class } & \multicolumn{2}{c}{ Training set } & \multicolumn{2}{c}{ Test set } \\
& Configuration & Number of samples & Configuration & Number of samples \\
\hline BMP2 & Sn_9563 & 233 & Sn_9566 & 196 \\
BTR70 & Sn_c71 & 233 & Sn_c71 & 196 \\
T72 & Sn_132 & 232 & Sn_812 & 196 \\
\hline
\end{tabular}

TABLE 6: Comparison of ARRs under configuration variance.

\begin{tabular}{lcccccc}
\hline Method & Proposed & SVM & SRC & CNN & MultiView1 & MultiView2 \\
\hline ARR (\%) & 98.84 & 94.92 & 95.08 & 97.35 & 97.97 & 98.14 \\
\hline
\end{tabular}

TABLE 7: Training and test sets under depression angle variance.

\begin{tabular}{lcccr}
\hline \multirow{2}{*}{ Class } & \multicolumn{2}{c}{ Training set } & \multicolumn{2}{c}{ Test set } \\
& Depression angle & Number of samples & Depression angle & Number of samples \\
\hline \multirow{2}{*}{ 2S1 } & & 299 & $30^{\circ}$ & 288 \\
& & & $45^{\circ}$ & 303 \\
BDRM2 & $17^{\circ}$ & 298 & $30^{\circ}$ & 287 \\
& & & $45^{\circ}$ & 303 \\
ZSU23/4 & 299 & $30^{\circ}$ & 288 \\
& & & $45^{\circ}$ & 303 \\
\hline
\end{tabular}

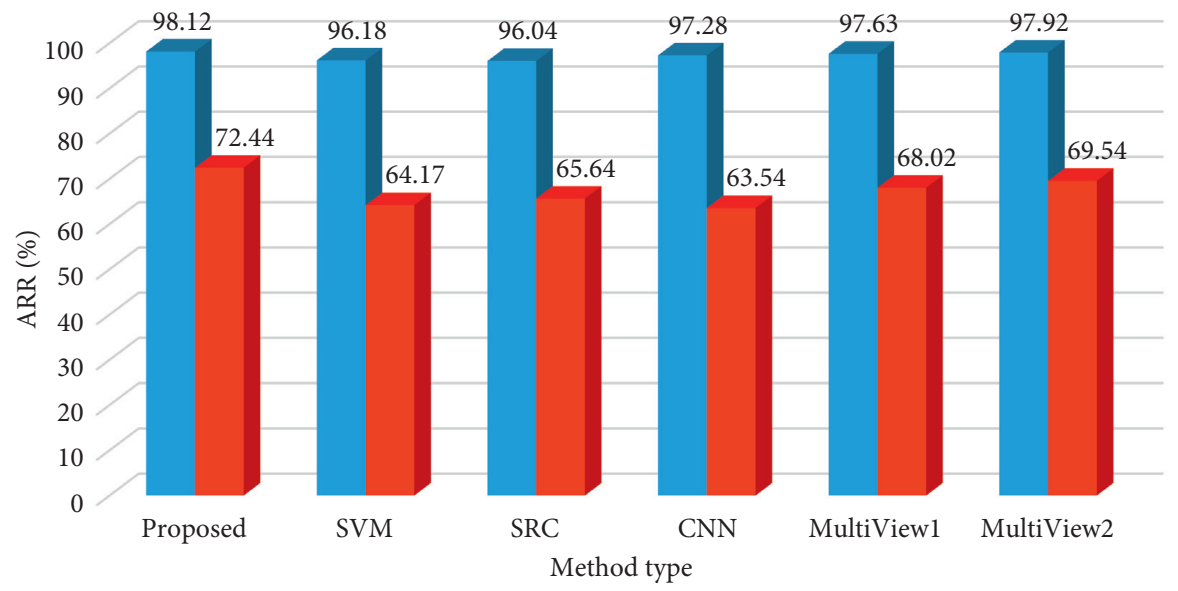

ㅁ $30^{\circ}$

- $45^{\circ}$

FIgURE 5: Comparison of ARRs at different depression angles.

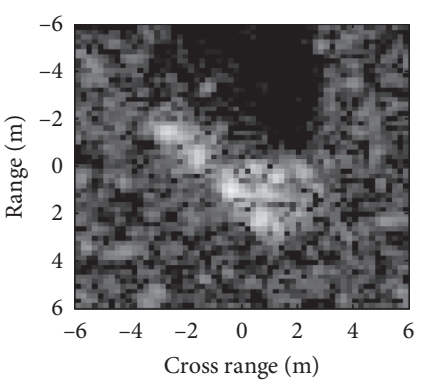

(a)

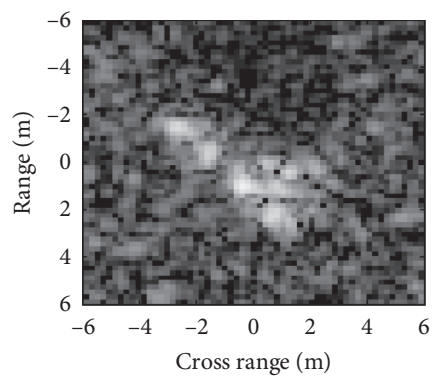

(b)

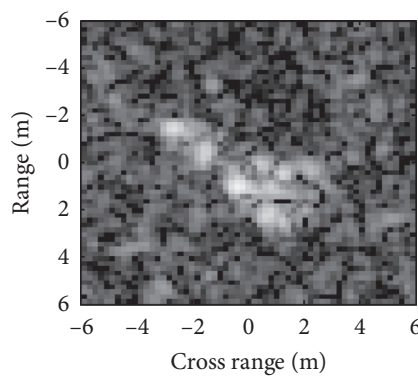

(c)

Figure 6: Continued. 


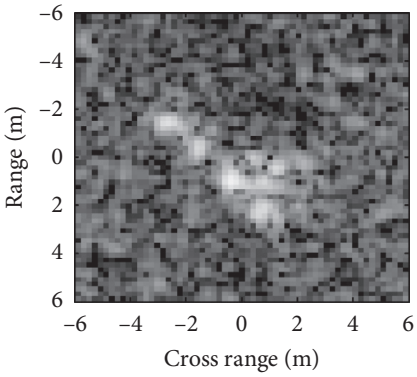

(d)

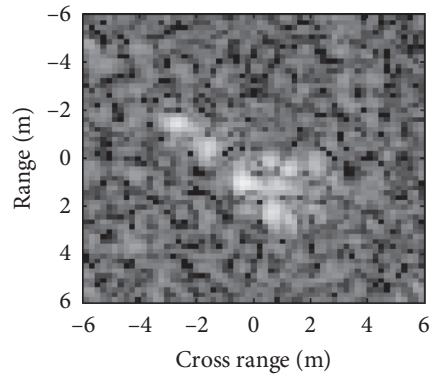

(e)

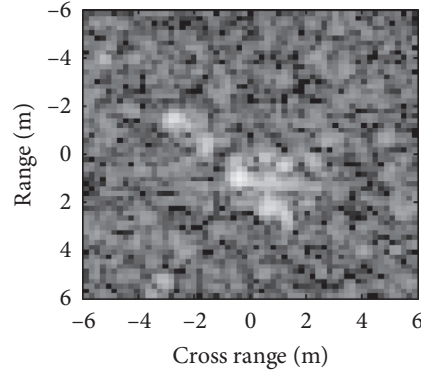

(f)

FiguRe 6: Illustration of simulated noisy SAR images at different levels. (a) Original. (b) $\mathrm{SNR}=10 \mathrm{~dB}$. (c) $\mathrm{SNR}=5 \mathrm{~dB}$. (d) $\mathrm{SNR}=0 \mathrm{~dB}$. (e) $\mathrm{SNR}=-5 \mathrm{~dB}$. (f) $\mathrm{SNR}=-10 \mathrm{~dB}$.

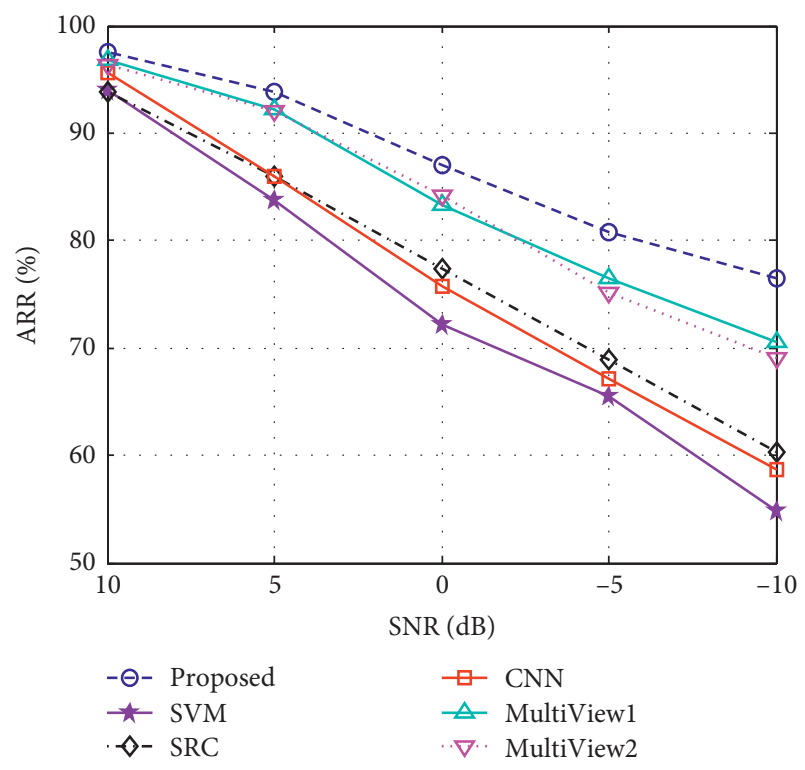

FIGURE 7: Comparison of ARRs at different depression noise levels.

\section{Conclusion}

In this paper, a multiview SAR target recognition method is proposed by considering both discrimination and correlation. SVM is first to classify each view thus rejecting those views with low contributions to correct decisions. A clustering algorithm is performed afterwards to conduct the correlation analysis. In each of the clustered view sets, the included SAR images are highly correlated. JSR is adopted to classify each view set and all the decisions are fused via a linear weighting strategy. Finally, the target label of an unknown test sample is determined based on the fused decision values. In the experiments, the proposed method is evaluated based on the MSTAR dataset under SOC and EOCs. Meanwhile, it is compared with some previous SAR target recognition methods including singleview and multiview ones. The results show that the proposed method could achieve much better performance than the compared ones.

\section{Data Availability}

The MSTAR dataset used to support the findings of this study is available online at http://www.sdms.afrl.af.mil/ datasets/mstar/.

\section{Conflicts of Interest}

The authors declare that they have no conflicts of interest.

\section{References}

[1] E. Khalid, W. G. Eric, M. Peter et al., "Automatic target recognition in synthetic aperture radar imagery: a state-ofthe-art review," Institute of Electrical and Electronics Engineers Access, vol. 4, pp. 6014-6058, 2016.

[2] C. Shan, B. Huang, and M. Li, "Binary morphological filtering of dominant scattering area residues for SAR target recognition," Computational Intelligence and Neuroscience, vol. 2018, Article ID 9680645, 15 pages, 2018.

[3] P. Bolourchi, H. Demirel, and S. Uysal, "Target recognition in SAR images using radial Chebyshev moments," Signal Image \& Video Processing, vol. 11, no. 6, pp. 1-8, 2017.

[4] B. Ding, G. Wen, J. Zhong et al., "Robust method for the matching of attributed scattering centers with application to synthetic aperture radar automatic target recognition," Journal of Applied Remote Sensing, vol. 10, no. 1, Article ID 016010, 2016.

[5] B. Ding, G. Wen, J. Zhong, C. Ma, and X. Yang, “A robust similarity measure for attributed scattering center sets with application to SAR ATR," Neurocomputing, vol. 219, pp. 130-143, 2017.

[6] A. K. Mishra, "Validation of PCA and LDA for SAR ATR," in Proceedings of the IEEE TENCON, pp. 1-6, Hyderabad, India, November 2008.

[7] Z. Cui, Z. Cao, J. Yang, J. Feng, and H. Ren, "Target recognition in synthetic aperture radar images via non-negative matrix factorisation," IET Radar, Sonar \& Navigation, vol. 9, no. 9, pp. 1376-1385, 2015.

[8] G. Dong, G. Kuang, N. Wang, L. Zhao, and J. Lu, "SAR target recognition via joint sparse representation of monogenic signal," Institute of Electrical and Electronics Engineers Journal of Selected Topics in Applied Earth Observations and Remote Sensing, vol. 8, no. 7, pp. 3316-3328, 2015.

[9] G. Dong and G. Kuang, "Classification on the monogenic scale space: application to target recognition in SAR image," 
Institute of Electrical and Electronics Engineers Transactions on Image Processing, vol. 24, no. 8, pp. 2527-2539, 2015.

[10] Q. Zhao and J. C. Principe, "Support vector machines for SAR automatic target recognition," Institute of Electrical and Electronics Engineers Transactions on Aerospace and Electronic Systems, vol. 37, no. 2, pp. 643-654, 2001.

[11] Y. Sun, Z. Liu, S. Todorovic, and J. Li, "Adaptive boosting for SAR automatic target recognition," Institute of Electrical and Electronics Engineers Transactions on Aerospace and Electronic Systems, vol. 43, no. 1, pp. 112-125, 2007.

[12] J. Wright, A. Y. Yang, A. Ganesh, S. S. Sastry, and Y. Yi Ma, "Robust face recognition via sparse representation," Institute of Electrical and Electronics Engineers Transactions on Pattern Analysis and Machine Intelligence, vol. 31, no. 2, pp. 210-227, 2009.

[13] J. J. Thiagaraianm, K. N. Ramamurthy, P. Knee, A. Spanias, and V. Berisha, "Sparse representations for automatic target classification in SAR images," in Proceedings of the 4th Int. Symp. Commun., Control Signal Process, pp. 1-4, Hong Kong, China, September 2010.

[14] H. Song, K. Ji, Y. Zhang, X. Xing, and H. Zou, "Sparse representation-based SAR image target classification on the 10-class MSTAR data set," Applied Sciences, vol. 6, no. 1, p. 26, 2016.

[15] S. Chen, H. Wang, F. Xu, and Y. Jin, "Target classification using the deep convolutional networks for SAR images," Institute of Electrical and Electronics Engineers Transaction on Geoscience and Remote Sensing, vol. 47, no. 6, pp. 1685-1697, 2016.

[16] J. Ding, B. Chen, H. Liu et al., "Convolutional neural network with data augmentation for SAR target recognition," Institute of Electrical and Electronics Engineers Geoscience and Remote Sensing Letters, vol. 13, no. 3, pp. 364-368, 2016.

[17] M. Z. Brown, "Analysis of multiple-view Bayesian classification for SAR ATR," in Proceedings of the SPIE, vol. 5095, pp. 265-274, Santa Fe, NM, USA, May 2003.

[18] R.-H. Huan and Y. Pan, "Target recognition for multi-aspect sar images with fusion strategies," Progress In Electromagnetics Research, vol. 134, pp. 267-288, 2013.

[19] H. Zhang, N. M. Nasrabadi, Y. Zhang, and T. S. Huang, "Multi-view automatic target recognition using joint sparse representation," Institute of Electrical and Electronics Engineers Transactions on Aerospace and Electronic Systems, vol. 48, no. 3, pp. 2481-2497, 2012.

[20] Z. Cao, L. Xu, and J. Feng, "Automatic target recognition with joint sparse representation of heterogeneous multi-view SAR images over a locally adaptive dictionary," Signal Processing, vol. 126, pp. 127-134, 2016.

[21] B. Ding and G. Wen, "Exploiting multi-view SAR images for robust target recognition," Remote Sensing, vol. 9, no. 11, p. 1150, 2017.

[22] J. A. Tropp, A. C. Gilbert, and M. J. Strauss, "Algorithms for simultaneous sparse approximation. Part II: convex relaxation," Signal Processing, vol. 86, no. 3, pp. 589-602, 2006.

[23] S. Ji, D. Dunson, and L. Carin, "Multitask compressive sensing," Institute of Electrical and Electronics Engineers Transactions on Signal Processing, vol. 57, no. 1, pp. 92-106, 2009.

[24] S. Doo, G. Smith, and C. Baker, "Target classification performance as a function of measurement uncertainty," in Proceedings of the 5th Asia-Pacific Conference on Synthetic Aperture Radar, pp. 587-590, Singapore, September 2015.

[25] Z. Zhang, "Joint classificaiton of multiresolution representaitons with discrimination anslusos for SAR ATR," Journal of Electronic Imaging, vol. 27, no. 4, Article ID 043030, 2018. 